\title{
MANUFACTURING PROCESS MANAGEMENT USING A FLEXIBLE MODELING AND SIMULATION APPROACH
}

\author{
Duilio Curcio \\ Francesco Longo \\ Giovanni Mirabelli \\ Modeling \& Simulation Center - Laboratory of Enterprise Solutions (MSC-LES) \\ Department of Mechanical Engineering, University of Calabria \\ Via Pietro Bucci, Rende, 87036, ITALY
}

\begin{abstract}
The focus of this paper is a manufacturing system placed in Calabria (Italy) which produces high pressure hydraulic hoses. The objective is to implement a simulator to be used both for carrying out specific analysis devoted to increase system efficiency and for supporting the manufacturing process management (implementation of all the features required for using real time the simulator).

After the modeling phase, the simulation model has been validated comparing simulation results with real system performances. In this paper the authors test tool potentials investigating system behavior under different scenarios obtained varying manufacturing departments configuration. The utilization degree of each manufacturing department and its daily production measure system performance.
\end{abstract}

\section{INTRODUCTION}

Oppositely to the analytical approaches, the importance of the Modeling \& Simulation approach on studying and analyzing manufacturing processes is related to the possibility to take into consideration several aspects of the process without introducing restrictive assumptions. Moreover a Modeling \& Simulation based approach allows to transfer easily the results obtained to the real system in order to evaluate its performance under different operative scenarios.

One of the most important advantages of Simulation is to explore and experiment possibilities for evaluating system behavior in correspondence of internal/external changes and for supporting process enhancement efficiency and organization (Bruzzone et al. 2006).

In effect the manufacturing process management (including internal material handling and logistics) is a quite complex task: consider, for instance, the a-priori planning of resources allocation, such planning must be updated as the time goes by for taking into consideration the stochastic variables that affect system processes and activities (job orders, flow of materials, machines, workstations, material handling systems, warehouses, etc.).

The state of art overview reports several studies in this specific field: McNaughton, (1959) faces the problem of scheduling with deadlines; Moore (1968) and Emmons (1969) respectively propose a simple study on one machine sequencing to minimize job lateness and job tardiness. Berry (1972) studies problems related to priority scheduling and inventory control of a production system. In effect, in manufacturing systems one of the most important problem is the production planning policy (see Nunnikhoven and Emmons 1977). According to literature, a great number of approaches for categorizing short period production planning problems have been proposed: Graves (1981) proposes a review of production scheduling approaches. Morito and Lee (1997) use simulation for dispatching rule optimization with random process time; Vaidyanathan and Park.(1998) use discrete event simulation in production scheduling. Moreover Longo et al. (2005) make a study about material flow analysis and plant lay-out optimization of a manufacturing system using a Modeling \& Simulation based approach and Longo et al. (2006) use Modeling \& Simulation for short period production planning in hydraulic hoses manufacturing.

In particular, the focus of this paper is to build a decision making tool, a simulator, to be used both for conducting specific analysis (e.g. what-if analysis) for process efficiency enhancement and for supporting the manufacturing process management (in this paper we aim at defining all the features to be implemented for using real time the simulator). Before getting into details, let us give a brief summary of the paper. Section 2 reports the description of the process for manufacturing hydraulic hoses. Section 3 presents the simulation model: as we will discuss later on, the modeling approach proposed by authors guarantees a flexible and time-efficient simulation model. Section 4 proposes the simulation model verification and validation; section 5 deals with the first application of the simulation 
model for investigating three different operative scenarios regarding one of the most important manufacturing system department. Finally the conclusions summarize critical issues and results of the paper.

\section{THE MANUFACTURING PROCESS}

As before mentioned, the production system considered in this paper (located in Calabria, south part of Italy) manufactures high pressure hydraulic hoses.

The plant belongs to a multinational company specialized in design, production and distribution of rubber hoses. The $L$-shape plant layout covers a surface of $13000 \mathrm{~m}^{2}$ and is subdivided into three operative sectors:

- mechanics;

- assembly;

- warehouses.

The mechanics sector manufactures ring nuts and junctions; it covers two different areas. The first one for cutting metallic sheets and the second one, starting from items coming from the previous working area, manufactures ring nuts and junctions.

In the assembly area, ring nuts, junctions, lock washers and hydraulic hoses are assembled.

The warehouse is also subdivided into two areas: the first one is the raw materials warehouse (rubber hoses, junction, ring nuts, etc.), the second one is the final products warehouse (assembled hydraulic hoses).

As we will explain in details in the next sections our simulation model takes into consideration only the assembly area.

\section{THE SIMULATION MODEL}

The research work presented in this paper has been developed under specific request of the company top management. The objective of the research study is twofold:

- the development of a tool to be used for carrying out what-if analysis (investigating different operative scenarios) and improving system performance.

- The same tool has to be used for the manufacturing process management (e.g. shop orders scheduling, inventory management, etc.);

After different meetings with the company top management we decided to implement a simulation model using the commercial package eM-Plant ${ }^{\mathrm{TM}}$ by Tecnomatix Technologies, a discrete event-oriented simulation tool.

The initial steps of the simulation study, problem formulation and setting of objectives (Banks, 1998), have highlighted two critical issues: (i) the simulation model flexibility (a model architecture capable of easily integrating future changes, in other words, the capability of recreating an evolving real system); (ii) the second critical issue regards the time for executing simulation runs (if the simulator has to be used for real time supporting the manufac- turing process management, we should have a timeefficient simulator).

To this end, we decided to choose (in using the software) a different modeling approach. In fact, the classical approach is based on library objects that can be used for reproducing static and dynamic entities. Figure 1 shows the material flow library provided by the software for modeling machines, workstations, conveyor, queues, transporters, etc.

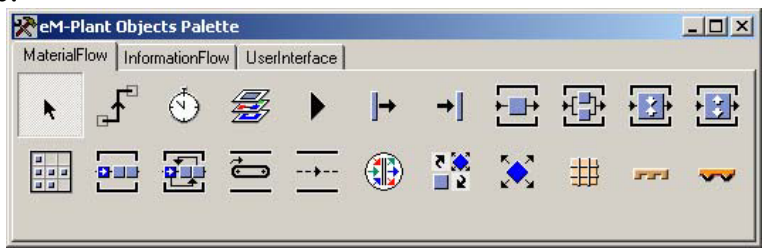

Figure 1: Material Flow Library

Such approach could give some problems in recreating the real system: sometimes the object provided by the software libraries are not capable of recreating the real system with satisfactory accuracy. In addition, the high number of dynamic entities flowing in the simulation model (consider that the real plant manufactures, on the average, 1000 hydraulic hoses per day) could increase the time required for executing a simulation run. In effect, consider that each entity defined in the simulation model is a class that requires memory until leaving the system (entity destruction); in other words the higher is the number of entities flowing in the simulation model, the higher is the memory required and the CPU utilization and the higher is the time required for executing a simulation run.

Our approach substitutes the flow of entities with a flow of information stored in tables and uses ad hoc programmed routines for implementing all the logics and rules governing the system. In fact, by using programming code to recreate the real scenario it becomes easy the implementation of future changes. To this end particular attention has been paid in creating model documentations and commenting the programming code. Usually the dynamic entities flowing in a discrete event simulation model determine the events lists evolution. In our modeling approach a flow of information takes the place of the flow of entities. Note that each dynamic entity is a class instanced in the model, thus, by deleting such classes we obtain a "light" simulator from a computational point of view. The library object "event generator" takes care of the events list management.

For a better understanding of the proposed approach let's focalize attention on the following situations. Consider for instance the simulation model initialization: we need to fill up the system with raw materials, components, shop orders in correspondence of each machine, etc. In the classical approach each shop order is an entity flowing in the model, in our approach each shop order is a row of a table in which are reported all the required information for a complete description of the shop order. The event list management is assured by event generator objects. Con- 
sider for instance the following events: "beginning of the shift at the cutting machine" and "beginning of the cutting operations related to a specific shop order". In the first case an event generator takes care of generating the event "beginning of the shift". In the second case the event management is more complex: a process time in a manufacturing system is usually a stochastic time, so at the "beginning of the of the cutting operation" we need to define the event of "cutting operation end". In this case by means of programming code and in correspondence of the "beginning" event we setup an event generator object that generates an event just after the stochastic process time is elapsed. Another method (programming code) could be used for recording in a table the information regarding the shop order worked at the cutting machine (i.e. shop order arrical time, shop order process time, etc.). This case of events management helps to understand how the programming code can be used for implementing all the rules governing the system and how programming code, event generators and tables interact for recreating the real system evolution.

The only disadvantage of this approach is the simulation model animation. The animation of a discrete event simulation model is strictly related to the flow of entities. By the way, in our case animation is not considered a priority aspect of the simulation study (however, the architecture of the model is ready for implementing the animation).

Such approach increases model flexibility and allows to obtain a great gain in terms of time required for executing a simulation run.

\subsection{Model Implementation}

The simulator main frame is called model. It contains 12 secondary frames. Each frame is built to recreate a specific process of the real system (assembly departments). The frames are named as follows:

- Gestore_Prod; it generates the production planning;

- Preparazione; it recreates the raw materials preparation;

- Marcatura; it recreates the fittings stamp operations;

- Taglio; it recreates the cutting operations;

- Spellatura; it recreates the hose skinning operations;

- Assemblaggio; it recreates the assembly operations;

- Pinzatura; it recreates the junction operations;

- Collaudo; it recreates the hoses testing operations;

- Controlli_Conf; it recreates the final controls and packaging operations;

- Prestazioni; it evaluates performance indexes values;

- Dialog; it implements the GUI (Graphic User Interface).
Note that most of the frames recreates the process required for manufacturing hydraulic hoses. Each secondary frame is now described in details. The Gestore_Prod implements all the rules and logics for production planning. In particular this object recreates customers' orders inserting, the inventory management, the raw materials allocation and the short period production planning. Its function is similar to the production planning office of the real system. Shop Orders (here in after S.O.s), generated at the beginning of the simulation and during the simulation by means of specific routines, are stored in tables. Note that in the classic modeling approach each S.O. is a dynamic entity (characterized by specific attributes) flowing in the model; in our approach each S.O. corresponds to a specific row of a table and the cell values corresponds to the entity attributes. The information flow is guaranteed by the "event generator" objects. In particular, in the Gestore_Prod frame we can distinguish three sections: S.O.s generation, inventory management and resources allocation. Figure 2 shows the frame architecture, note that there are only three different type of library objects: "Tables" for storing information (see the first object in the top-left corner), "Methods" for programming code (see the M-shape icons) and "Event Generators" for events lists management (see the second object in the top-left corner).

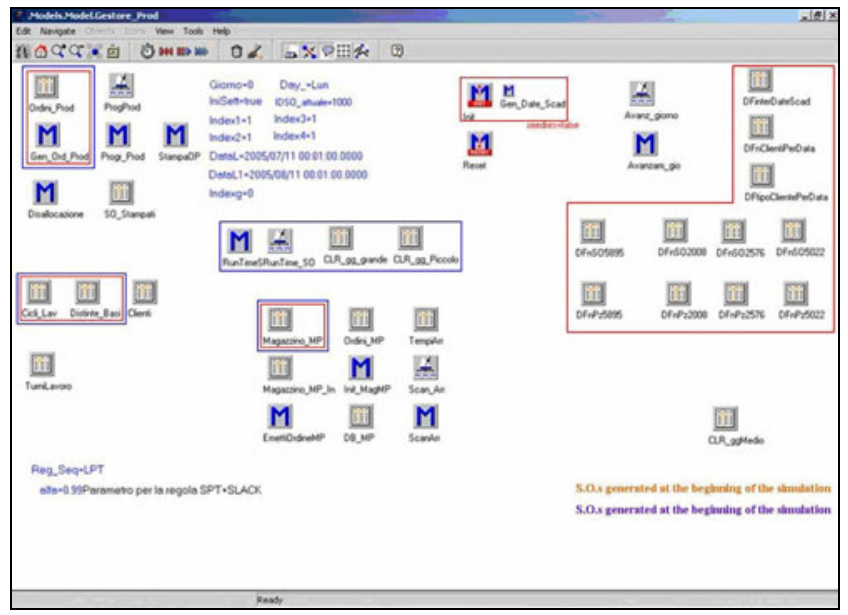

Figure 2: Modeling frame for production planning

The Preparazione modeling frame implements all the rules related to the first operation of the job routing, the raw materials preparation. The worker takes the S.O.s raw materials from warehouse shelves and sorts them according to the scheduling rules suggested by the production planning office. As in the previous case only three types of library objects forms the frame architecture opportunely subdivided in three sections: the first section for collecting and scheduling S.O.s; the second for defining the specific manufacturing operations associated to each S.O; the third for collecting performance indexes values during the simulation. 
The remaining frames have the same architecture of the previous one. Only the Taglio modeling frame (that recreates cutting operations, see Figure 3) implements specific features. In the actual configuration workers manually execute cutting operations. As required by the company top management the simulator should also be used for evaluating system performance under different cutting scenarios. To this end, in addition to the manual operation, an automated cutting process has been implemented. The results of such analysis will be discussed later on in the paper.

Note that each manufacturing system department has the same modeling architecture for guarantying high flexibility in terms of future changes implementation.

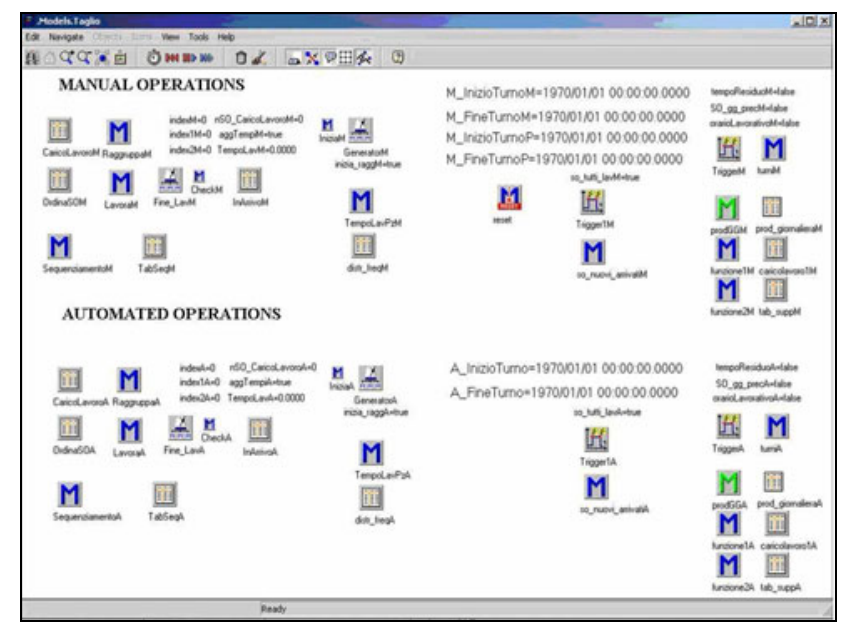

Figure 3: Modeling Frame for cutting operations

The Prestazioni modeling frame collects performance indexes daily values during the simulation (such as average flow time, average lateness, average tardiness, department utilization degrees, department production). Note that performance indexes have been selected both to use the simulator for analyzing different operative scenarios (as we will see for the cutting departments) and for supporting the manufacturing process management (e.g. S.O.s scheduling).

Finally the Dialog frame implements the graphic user interface for setting system parameters before starting the simulation (e.g. S.O.s scheduling rules, simulation length etc.).

\subsection{Input Data}

The simulator is directly connected with the company informative system by means of MS Excel spreadsheets. The company informative system maintains the spreadsheets updated with historical data of the last six months, such as:

- products;

- customers;

- process times;

- stocks and refurbishment times;

- due dates;
- frequency of customers requiring orders;

- frequency of customer orders;

- number of S.O.s for each customer;

- quantity for each S.O.

The MS Excel spreadsheets have been organized for calculating empirical distribution starting from input data. The simulator receives as input the empirical distributions (see Figure 4 for the empirical distribution of customers number per date).

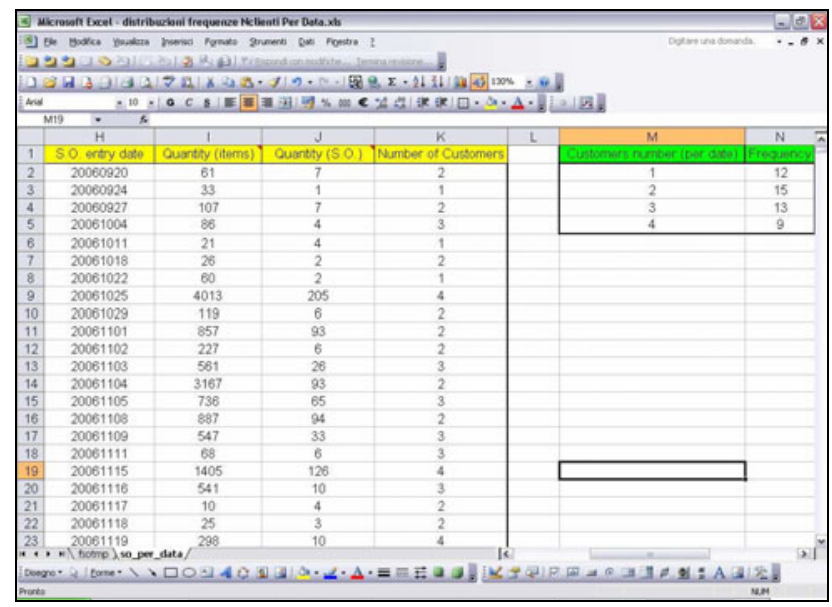

Figure 4: Customers number per date, empirical distribution

\section{MODEL VERIFICATION AND VALIDATION}

Verification is the process of determining that a model implementation accurately represents developer conceptual description and specifications (Balci, 1998).

The simulation model verification has been made using the debugging technique. The model has been debugged, following an iterative procedure, for finding and eliminating all the bugs due to model translation.

Validation is the process of determining the degree to which a model is an accurate representation of the real world from the perspective of the intended use of the model (Balci, 1998).

The data used for validating the simulation model regard an historical period from January 2006 to December 2006.

The validation process is made up by two different steps:

- evaluation of the simulation run length;

- validation using the Face Validation technique.

\subsection{Simulation Run Length}

The simulation run length is usually the first step of the validation process. A simulation run must be long enough to guarantee reliable statistic results; a longer simulation run doesn't give additional information and requires more time to be executed. 
To evaluate the simulation run length we use the Mean Square Pure Error analysis $\left(\mathrm{MS}_{\mathrm{PE}}\right)$ considering as performance index the plant mean daily production. As reported in Figure 5, after 160 days the value of the $\mathrm{MS}_{\mathrm{PE}}$ of the plant mean daily production is small enough for assuring the goodness of the simulation model statistic results. The value of the Mean Square pure Error has been used for calculating a confidence interval and such confidence interval has been compared with the confidence interval obtained by real data. The confidence intervals are quite similar so we can conclude that 160 days is the optimal simulation run length.

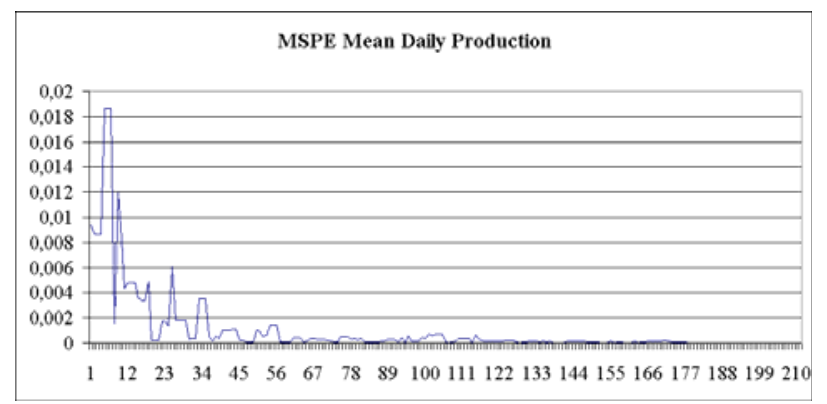

Figure 5: MSpE Analysis

\subsection{Validation}

As before mentioned the validation of the simulation model has been conducted using the Face Validation method. Potential users of the model, system's experts, compare model and system behaviors under identical input conditions and judge whether the model and its results are reasonable (Balci, 1998). Also in this case the performance index considered is the mean daily production of each workstation.

Each simulation run has been replicated 5 times. In order to neglect the effects due to the simulator warm-up period, we don't consider the initial values of the real performance index.

Figure 6 shows the mean daily production for the assembly operation. The graph was shown to company experts asking to make the difference between real and simulated curves. In effect, the curves are pretty similar and the experts were unable to make such difference, thus, we conclude that the model is an accurate representation of the real system. Note that similar results have been obtained for each department.

\section{WHAT-IF ANALYSIS: THREE DIFFERENT CUT DEPARTMENT SCENARIOS}

As mentioned earlier, the simulation model should be used for carrying out specific what-if analysis and for supporting the manufacturing process management and. In this paper we propose the first application of the simulator for in- vestigating three different operative scenarios concerning the cut department.

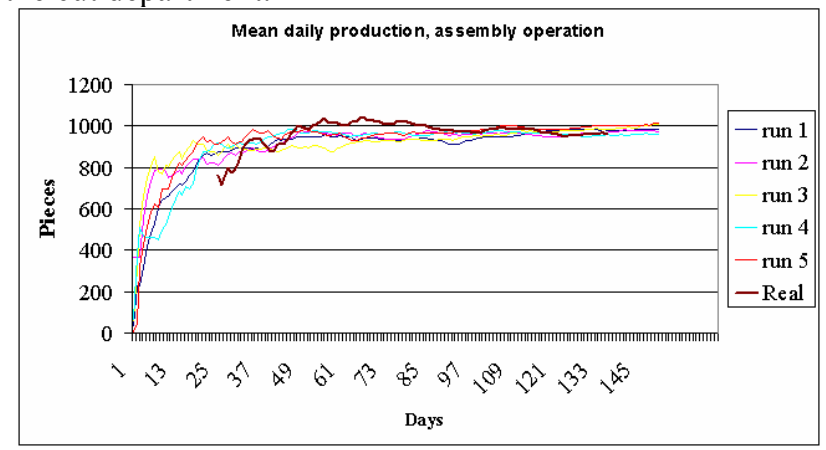

Figure 6: Mean daily production for Assembly Operation (real and simulated)

Note that the simulation model architecture has been developed including all the required features for using real time the simulator for supporting the manufacturing process (flexibility, time efficient, interface with the company informative system, etc.).

The three different scenarios being considered are:

- manual operated cut (actual configuration, scenario 1);

- $\quad$ automatic operated cut (scenario 2);

- manual and automatic operated cut (Scenario 3).

Let us emphasize that simulation results reported in this section should be analyzed by the company top management taking into consideration both technical efficiency and economic aspects. In fact, Scenario 2 obviously requires an higher amount of money for buying a completely automated machine capable of respecting the plant mean daily production.

We take into consideration the following factors for estimating system performance:

- daily production of each productive department;

- utilization degree of each productive department.

The total number of simulation runs is 3 , one simulation run for each scenario. Each simulation run has been replicated 5 times, so the total number of replications is 15 $(5 \times 3=15)$. Each replication has a length of 160 days as evaluated by the Mean Square Pure Error analysis. Note that each replication requires about 2 minutes. Such low time is essentially due to the simulator architecture (absence of dynamic entities flowing in the model). Thanks to this approach the simulator can be easily used real time for supporting system management.

\subsection{Simulation results analysis}

Tables 1 and 2 consist of simulation results in terms of degree utilization and daily production of each department. From simulation results analysis the best scenario is the third one characterized by an average utilization degree of 0,75 and an average daily production of 844 units (such 
values are referred to the entire plant). However there is a slightly difference between the second and the third scenario. The third scenario performs better because for some types of hydraulic hoses the manual cut requires a smaller process time, thus a mixed approach (manual and automated) gives better results. As mentioned earlier the second scenario requires an high amount of money for buying the automated machine. Further considerations regards the number of workers. In the actual scenario there are 2 workers, the second scenario requires one worker and the third scenario requires, once again, 2 workers. Thus the higher amount of money in the third scenario could be balanced in the long period by lower manpower costs.

Table 1: Utilization degrees for each scenario

\begin{tabular}{lccc}
\hline Department & Scen.1 & Scen.2 & Scen.3 \\
\hline Preparation & 0,80 & 0,81 & 0,80 \\
Fittings stamp & 0,87 & 0,87 & 0,86 \\
Manual Cut & 0,97 & 0,00 & 0,81 \\
Automated Cut & 0,00 & 0,97 & 0,71 \\
Skinning operation & 0,56 & 0,86 & 0,89 \\
Assembly & 0,42 & 0,66 & 0,67 \\
Junction & 0,45 & 0,69 & 0,70 \\
Testing operations & 0,48 & 0,73 & 0,74 \\
Packaging & 0,44 & 0,63 & 0,63 \\
\hline Average Value & $\mathbf{0 , 5 5}$ & $\mathbf{0 , 6 9}$ & $\mathbf{0 , 7 5}$ \\
\hline
\end{tabular}

Table 2: Daily production for each scenarios

\begin{tabular}{lccc}
\hline Department & Scen.1 & Scen.2 & Scen.3 \\
\hline Preparation & 931 & 933 & 927 \\
Fittings stamp & 975 & 967 & 956 \\
Manual Cut & 510 & 0 & 386 \\
Automated Cut & 0 & 994 & 641 \\
Skinning operation & 385 & 697 & 703 \\
Assembly & 507 & 974 & 986 \\
Junction & 508 & 971 & 1003 \\
Testing operations & 512 & 974 & 1002 \\
Packaging & 519 & 980 & 998 \\
\hline Average Value & $\mathbf{5 3 8}$ & $\mathbf{8 3 2}$ & $\mathbf{8 4 4}$ \\
\hline
\end{tabular}

Figures 7-8 graphically reports simulation results for two different departments.

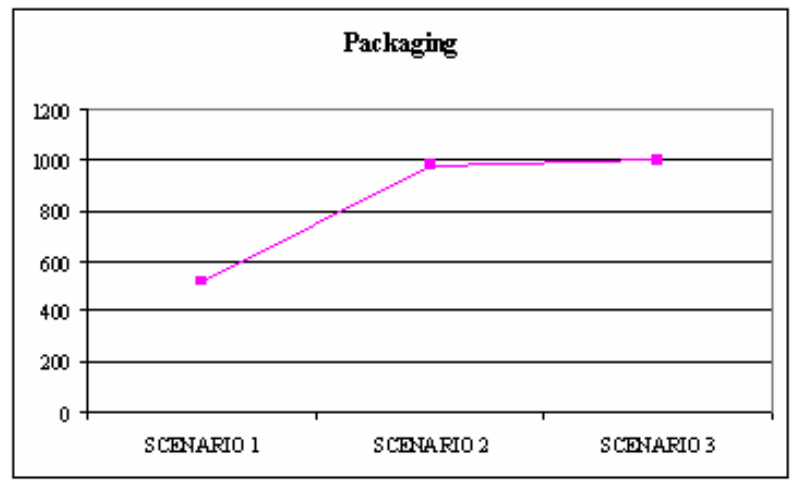

Figure 7: Utilization degree for final controls operations

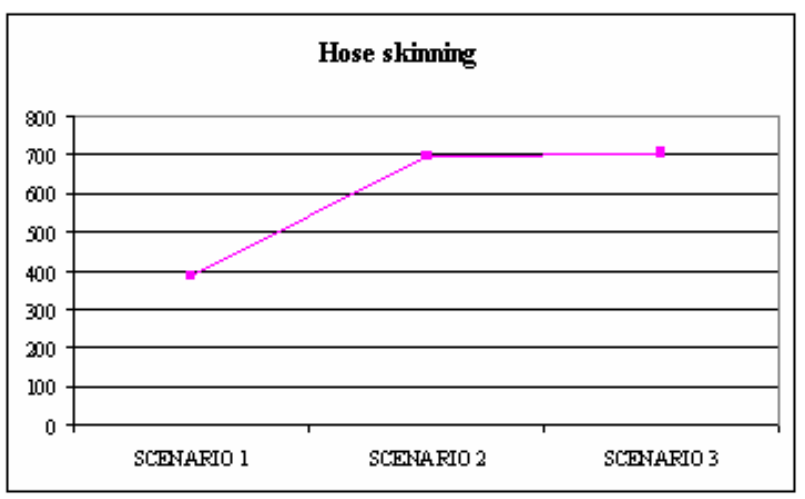

Figure 8: Daily production for hoses skinning operations

We do not give any results concerning the final choice of the company top management; the objective of this paper is to present simulator architecture. We proposed the what-if analysis regarding the cut department for answering to a specific request of the company and for showing simulator potentials.

\section{CONCLUSIONS}

The simulation model recreates, with high flexibility, all the processes and operations for manufacturing hydraulic hoses. The modeling approach (a discrete event approach) based on tables, programming code and event generators performs better than the traditional approach (based library objects) in terms of model flexibility (capability of recreating an evolving real system), of time and computational resources required for executing a simulation run. The simulation model validation (performed according to real data) shows the capability of the simulation model to recreate with satisfactory accuracy the real system. Hence, we conclude that the simulator can be used for carrying out specific what-if analysis (system efficiency enhancement) and could be used for supporting (real time) the manufacturing process management.

The what-if analysis proposed in this paper shows simulator potentials in investigating different operative scenarios. In particular the analysis carried out with the 
simulation model investigates system utilization degree and daily production under different cut departments scenarios. Simulation results shows a slight difference in terms of utilization degrees or daily production between the second and the third scenario, thus, the only discrimination parameter is the economic one.

\section{REFERENCES}

Banks, J. 1998. Handbook of simulation. Georgia Institute of Technology, Georgia: John Wiley \& Sons, Inc.

Balci, O. 1998. Verification, Validation and Testing. In Handbook of Simulation, J. Banks eds., pp.335-393, Georgia: John Wiley \& Sons, Inc.

Berry, W.L. 1972. Priority scheduling and inventory control in a job shop lot manufacturing systems. AIIE Transactions 4: 267-276.

Bruzzone, A.G., M.Brandolini and F.Longo. 2006. Enhancement process based on simulation supporting efficiency \& Organization. In Proceedings of SCSC ( July 30 - August 03). Calgary, CND.

Emmons, H.1969. One machine sequencing to minimize certain functions of job tardiness. Operational Research: 701-715.

Graves, C. G. 1981. A review of production scheduling. Operations Research: 646-675.

Longo F., G. Mirabelli and E. Papoff. 2005. Material Flow Analysis and Plant Lay-Out Optimization of a Manufacturing System. International Journal of Computing, No. 1, issue 5, 107-116.

Longo F., G. Mirabelli and E. Papoff. 2006. Modeling, analysis \& simulation of tubes manufacturing process an industrial operations controls. In Proceedings of SCSC ( July 30 - August 03). Calgary, CND.

McNaughton, R. 1959. Scheduling with deadlines and loss functions. Management Science 1: 1-12.

Moore, J.M. 1968. An n job, one machine sequencing algorithm for minimizing the number of late jobs. Management Science 1: 102-109.

Morito, S., Lee K.H.1997. Efficient simulation optimization of dispatching priority with random processing time. Proceedings of 1997 Winter simulation Conference.

Nunnikhoven, T.S., Emmons H. 1977. Scheduling on parallel machines to minimize two criteria related to job tardiness. AIIE Transactions 3: 288-296.

Vaidyanathan, B.S., Y. H. Park.1998. Application of discrete event simulation in production scheduling. Proceedings of 1998 Winter simulation Conference.

\section{AUTHOR BIOGRAPHIES}

DUILIO CURCIO was born in Vibo Valentia (Italy), on December the $15^{\text {th }}, 1981$. He took the degree in Mechanical Engineering from University of Calabria (2006). He is cur- rently PhD student at the Mechanical Department of University of Calabria. His e-mail address is <dcurcio@unical.it>.

FRANCESCO LONGO was born in Crotone (Italy), on February the $08^{\text {th }}, 1979$. He took the degree in Mechanical Engineering from University of Calabria (2002). He received the PhD in Industrial Engineering (2005). He is currently researcher at the Mechanical Department (Industrial Engineering Section) of University of Calabria and scientific responsible of the Modeling \& Simulation Center Laboratory of Enterprise Solutions (MSC-LES) in the same department. His research interests regard modeling \& simulation of manufacturing systems and supply chain, DOE, ANOVA. His e-mail address is $<$ f.longo@unical.it $>$.

GIOVANNI MIRABELLI was born in Rende (Italy), on January the $24^{\text {th }}, 1963$. He took the degree in Industrial Technology Engineering from University of Calabria. He is currently researcher at the Mechanical Department (Industrial Engineering Section) of University of Calabria. His research interests regard work measurement and human reliability. His e-mail address is $<$-mirabellieunical.it $>$. 